\title{
Distribution of the Population at Risk of Cholangiocarcinoma in Bua Yai District, Nakhon Ratchasima of Thailand Using Google Map
}

\author{
Soraya J Kaewpitoon ${ }^{1,2,3 *}$, Ratana Rujirakul ${ }^{1}$, Amnat Sangkudloa ${ }^{4}$, Sarochinee \\ Kaewthani $^{5}$, Kritsakorn Khemplila ${ }^{6}$, Karuna Cherdjirapong ${ }^{7}$, Jirawoot \\ Kujapun $^{8}$, Jun Norkaew ${ }^{8}$, Wasugree Chavengkun ${ }^{8}$, Sukanya Ponphimai ${ }^{8}$, \\ Poowadol Polsripradist ${ }^{9}$, Natnapa Padchasuwan ${ }^{10}$, Apinya Joosiri ${ }^{1}$, Parichart \\ Wakkhuwattapong ${ }^{1}$, Ryan A Loyd ${ }^{2}$, Likit Matrakool ${ }^{3}$, Taweesak Tongtawee ${ }^{3}$, \\ Sukij Panpimanmas ${ }^{3}$, Natthawut Kaewpitoon ${ }^{1,3,8}$
}

\begin{abstract}
Background: Cholangiocarcinoma (CCA), a major problem of health in Thailand, particularly in Northeastern and Northern regions, is generally incurable and rapidly lethal because of presentation in stage 3 or 4 . Early diagnosis of stage 1 and 2 could allow better survival. Therefore, this study aimed to provide a distribution map of populations at risk for CCA in BuaYai district of Nakhon Ratchasima province, Northeast Thailand. Materials and Methods: A cross-sectional survey was carried out in 10 sub-districts and 122 villages, during June and November 2015. The populations at risk for CCA were screened using the Korat CCA verbal screening test (KCVST) and then risk areas were displayed by using Google map (GM). Results: A total of 11,435 individuals from a 26,198 population completed the KCVST. The majority had a low score of risk for CCA (1-4 points; 93.3\%). High scores with 6,7 and 8 points accounted for $1.20 \%, 0.13 \%$ and $0.02 \%$. The population at risk was found frequently in sub-district municipalities, followed by sub-district administrative organization and town municipalities, $(\mathrm{F}=396.220$, P-value=0.000). Distribution mapping comprised 11 layers: 1, district; 2, local administrative organization; 3, hospital; 4, KCVST opisthorchiasis; 5, KCVST praziquantel used; 6, KCVST cholelithiasis; 7, KCVST raw fish consumption; 8, KCVST alcohol consumption; 9, KCVST pesticide used; 10, KCVST relative family with CCA; and 11, KCVST naïve northeastern people. Geovisual display is now available online. Conclusions: This study indicated that the population at high risk of CCA in Bua Yai district is low, therefore setting a zero model project is possible. Key success factors for disease prevention and control need further study. GM production is suitable for further CCA surveillance and monitoring of the population with a high risk score in this area.
\end{abstract}

Keywords: Distribution - population at risk - cholangiocarcinoma - Thailand - Google map

Asian Pac J Cancer Prev, 17 (3), 1433-1436

\section{Introduction}

Cholangiocarcinoma (CCA) is a bile duct cancer, which originates in biliary epithelial cells, and occurs in the intrahepatic and extrahepatic regions of the bile duct (Green et al., 1991; Khuntikao 2005; Bhudhisawadi et al., 2012). CCA is known to be one of the most common causes of cancer related to death in Thailand where is the highest incident of the world (Green et al., 1991; Sripa et al., 2007; Shin et al., 2010). CCA is considered to be an incurable and rapidly lethal disease unless all the tumors can be fully resected. Three-year survival rates of $35 \%$ to $50 \%$ are achieved only in a subset of patients who have negative histological margins at the time of surgery (Akamatsu et al., 2011). Mortality rate of liver cancer rate in different regions of Thailand has been reported

${ }^{1}$ Parasitic Disease Research Unit, ${ }^{2}$ School of Family Medicine and Community Medicine, ${ }^{3}$ Suranaree University of Technology Hospital, Suranaree University of Technology, Nakhon Ratchasima, ${ }^{4}$ Geographic Information Technology Program, Faculty of Information Technology, Rajabhat Mahasarakham University, Maha Sarakham, ${ }^{5}$ Geo-Informatics Program, Faculty of Science and Technology, ${ }^{6}$ Environmental Technology Program, ${ }^{7}$ Public Administration Program, Faculty of Humanities and social Science, Nakhon Ratchasima Rajabhat University, ${ }^{8}$ Faculty of Public Health, Vongchavalitkul University, ${ }^{9}$ Nakhon Ratchasima Provincial Public Health Office, Nakhon Ratchasima, ${ }^{10}$ Faculty of Public Health, Khon Kaen University, Khon Kaen, Thailand $*$ For correspondence: soraya.k@sut.ac.th 
and found that Nakhon Ratchasima province has 13.6716.2 per 100,000 populations (Sripa et al., 2008). Early diagnosis of stage 1 and 2 could be encourage the patients have a long life.

Surveillance and distribution of the population at risk for CCA are need required. We previously have been applied the geographic information sytem and Google EarthTM for distribution mapping of CCA and liver fluke infection, and found that the Google map (GM) production process is very simple and easy to learn. It is suitable for the user in the further CCA management strategy (Rattanasing et al., 2015). The combination of GIS and statistical analysis can help simulate the spatial distribution and risk areas of liver fluke, and thus may be an important tool for future planning of prevention and control measures (Rujirakul et al., 2015). Therefore, this study aimed to investigate the population at risk for CCA in BuaYai district of Nakhon Ratchasima province, Northeast of Thailand, and the distribution mapping of risk areas by using GM.This data is useful for further surveillance, monitoring, and long term care for the high risk areas.

\section{Materials and Methods}

\section{Study design and location}

A cross-sectional survey was conducted in BuaYai district, Nakhon Ratchasima province, northeastern Thailand, between June and November 2015. Bua Yai is a district in the northern part of Nakhon Ratchasima, and neighboring districts are (from the north clockwise) Waeng Noi district of KhonKaen province, Bua Lai, Sida, Non Daeng, Khong, Ban Lueam, and Kaeng Sanam Nang of Nakhon Ratchasima Province. Bua Yai district is subdivided into 10 sub-districts, and 121 villages. The district is sub-divided into local administrative organization including Town Municipality (BuaYai town), Sub-district Municipality (Nong Pluang and Khu Khad), and Sub-district Administrative Organization (Don Chum Chang, Dong Bang, Don KhonTha, Nong Chaeng Noi, Khok Sa-ad, Nong Chaeng Yai, Khok Si, Nong Chaeng
Ko, Non Thong Lang, BuaYai, and Thong Lang Noi). This district is coverage areas $271.6 \mathrm{~km} 2$ (104.9 sq mi), and has 84,133 populations. The study protocol was approved by Suranaree University Ethical Review Committee, EC58-48.

\section{Study population and data collection}

A total of 11,435 populations from 26,198 populations who have age over 35 years old, was purposive selected and completed the verbal screening. The majorities of population were female (56.40\%), age group 51-60 years old $(30.00 \%)$, habited in Sub-district Administrative Organization (SAO) $(76.54 \%)$ (Table1).Populations at risk for CCA, were screened by using Korat-CCA Verbal Screening Test: KCVST, contained general information (name, gender, age, address, and health catchment area), and the question (yes/no choices) of the history with (1) opisthorchiasis, (2) praziquantel used, (3) cholelithiasis, (4) raw fish consumption, (5) alcohol consumption, (6) pesticide used, (7) relative family with CCA, and (8) naïve northeastern people. Before the study, permission and collaboration of the head of the public health center and/or head of the sub-district were obtained.

\section{Software and Management}

Microsoft excel, ArcGIS 10.2, and Google map were used in this study. Spatial database was contained thepoints of sub-district boundaries, catchment areas of local administration and hospital, anddistrict boundaries. Attribute database was contained the populations at risk for CCA; no risk, 1-4, 5, 6, 7, and 8 scores. Frequency of scores of each question from KCVST and the type of local administrative organization was calculated according Table 2. Data were tranfered to WGS84 UTM ZONE 48. The point with the populations at risk for CCA,was used to analyze and calculate with ArcGIS 10.2. After the conversion, all of the data were imported into GM using online web pages www.earthpoint.us. This web pages using HTML codes (village point was a primary key) to export the coordinate to $\mathrm{kml}$ format that can be recognized by GM.

Table 1. Participants in Bua Yai district, Nakhon Ratchasima Province, Thailand, who had Screened by using KVST, and Classified by Gender, age, and Catchment Areas

\begin{tabular}{|c|c|c|c|c|c|c|c|c|}
\hline \multirow[t]{3}{*}{ Characteristics } & \multicolumn{6}{|c|}{ Catchment areas } & \multicolumn{2}{|c|}{ Total } \\
\hline & \multicolumn{2}{|c|}{$\mathrm{TM}$} & \multicolumn{2}{|c|}{ SM } & \multicolumn{2}{|c|}{ SAO } & & \\
\hline & $\mathrm{n}$ & $\%$ & $\mathrm{n}$ & $\%$ & $\mathrm{n}$ & $\%$ & $\mathrm{n}$ & $\%$ \\
\hline Gender & 1,951 & 100 & 991 & 100 & 8,493 & 100 & 11,435 & 100 \\
\hline Male & 833 & 42.70 & 424 & 42.79 & 3728 & 43.89 & 4985 & 43.59 \\
\hline Female & 1,118 & 57.30 & 567 & 57.21 & 4762 & 56.07 & 6447 & 56.38 \\
\hline No data & 0 & 0.00 & 0 & 0.00 & 3 & 0.04 & 3 & 0.03 \\
\hline \multicolumn{9}{|c|}{ Fisher's Exact Test $=1.667, \mathrm{p}=0.748$} \\
\hline Age & 1,951 & 100 & 732 & 100 & 8,752 & 100 & 11,435 & 100 \\
\hline $35-40$ & 242 & 12.40 & 116 & 11.71 & 860 & 10.13 & 1,218 & 10.65 \\
\hline $41-50$ & 509 & 26.09 & 280 & 28.25 & 2,480 & 29.20 & 3,269 & 28.59 \\
\hline $51-60$ & 532 & 27.27 & 299 & 30.17 & 2,594 & 30.54 & 3,425 & 29.95 \\
\hline $61-70$ & 435 & 22.30 & 209 & 21.09 & 1,676 & 19.73 & 2,320 & 20.29 \\
\hline$>70$ & 233 & 11.94 & 87 & 8.78 & 883 & 10.40 & 1,203 & 10.52 \\
\hline
\end{tabular}

TM: Town Municipality, SM: Sub-district Municipality, SAO: Sub-district Administrative Organization 
Table 2. Percentage of Participant Answer Each Item of Korat Verbal Screening Test

\begin{tabular}{|c|c|c|c|c|c|c|c|c|c|}
\hline \multirow{2}{*}{ Sub-district hospital and catchment areas } & \multirow{2}{*}{$\mathrm{n}$} & \multicolumn{8}{|c|}{ Item list of Korat-verbal screening test } \\
\hline & & $\mathrm{Ov}$ & PZQ & CLT & RFC & $\mathrm{AC}$ & PU & RFCCA & NNE \\
\hline Town Municipality & 1,951 & 1.08 & 1.74 & 1.08 & 52.33 & 36.8 & 17.89 & 2.10 & 87.49 \\
\hline Bua Yai town & 1,951 & 1.08 & 1.74 & 1.08 & 52.33 & 36.8 & 17.89 & 2.10 & 87.49 \\
\hline Sub district Municipality & 991 & 4.14 & 7.97 & 2.22 & 95.76 & 69.22 & 71.14 & 1.72 & 98.39 \\
\hline Nong Phluang & 365 & 0.82 & 0.82 & 1.10 & 100 & 75.34 & 50.68 & 1.37 & 99.73 \\
\hline Khu Khad & 626 & 6.07 & 12.14 & 2.88 & 93.29 & 65.65 & 83.07 & 1.92 & 97.6 \\
\hline Sub district Administrative Organization (SAO) & 8,493 & 4.24 & 7.91 & 1.30 & 86.47 & 46.79 & 64.41 & 2.47 & 97.46 \\
\hline Don Chum Chang & 530 & 0.94 & 8.87 & 1.32 & 97.92 & 47.17 & 70.57 & 1.51 & 97.92 \\
\hline Dong Bang & 1,105 & 9.05 & 8.14 & 1.45 & 95.84 & 61 & 87.33 & 3.35 & 97.8 \\
\hline Don Khon Tha & 658 & 2.43 & 6.53 & 1.22 & 94.53 & 60.18 & 72.49 & 2.13 & 98.78 \\
\hline Nong Chaeng Noi & 804 & 1.74 & 5.1 & 0.37 & 92.41 & 43.41 & 74.88 & 1.87 & 98.76 \\
\hline Khok Sa-ad & 1071 & 2.99 & 6.44 & 0.84 & 91.97 & 48.27 & 46.5 & 2.05 & 98.6 \\
\hline Nong Chaeng Yai & 1,000 & 2.9 & 5.5 & 1.3 & 89.5 & 46.9 & 66.3 & 3 & 98.1 \\
\hline Khok Si & 641 & 5.3 & 7.96 & 2.65 & 81.9 & 37.75 & 58.03 & 2.5 & 97.97 \\
\hline Nong Chaeng Ko & 853 & 7.85 & 19.34 & 2.34 & 81.59 & 39.27 & 59.79 & 1.88 & 96.25 \\
\hline Non Thong Lang & 859 & 1.63 & 7.45 & 0.93 & 80.21 & 39.58 & 51.8 & 4.07 & 95.23 \\
\hline Bua Yai & 251 & 4.78 & 8.37 & 0.4 & 66.53 & 48.21 & 56.18 & 3.19 & 90.84 \\
\hline Thong Lang Noi & 676 & 5.47 & 3.85 & 1.18 & 65.53 & 41.57 & 62.57 & 1.33 & 97.04 \\
\hline Total & 11,435 & 3.69 & 6.86 & 1.34 & 81.45 & 47.03 & 57.05 & 2.34 & 95.84 \\
\hline
\end{tabular}

Ov; Opisthorchiasis, PZQ; praziquantel used, CLT; cholelithiasis, RFC; raw fish consumption, AC; alcohol consumption, PU; pesticide used, RFCCA; relative family with CCA, NNE; naive Northeastern people

Table 3. Risk Score of Participant who were Screened by Using the Korat Verbal Screening Test

\begin{tabular}{|c|c|c|c|c|c|c|c|}
\hline \multirow[t]{2}{*}{ Catchment Areas } & \multicolumn{7}{|c|}{ Risk Score } \\
\hline & 0 point & 1-4 point & 5 points & 6 points & 7 points & 8 points & Total \\
\hline \multicolumn{8}{|l|}{ TM } \\
\hline Count & 127 & 1799 & 13 & 7 & 4 & 1 & 1951 \\
\hline$\%$ within area & 6.51 & 92.21 & 0.67 & 0.36 & 0.21 & 0.05 & 100.00 \\
\hline \multicolumn{8}{|l|}{ SM } \\
\hline Count & 0 & 904 & 62 & 23 & 1 & 1 & 991 \\
\hline$\%$ within area & 0.00 & 91.22 & 6.26 & 2.32 & 0.10 & 0.10 & 100.00 \\
\hline \multicolumn{8}{|l|}{ SAO } \\
\hline Count & 47 & 7966 & 363 & 107 & 10 & 0 & 8493 \\
\hline$\%$ within area & 0.55 & 93.80 & 4.27 & 1.26 & 0.12 & 0.00 & 100.00 \\
\hline \multicolumn{8}{|l|}{ Total } \\
\hline Count & 174 & 10669 & 438 & 137 & 15 & 2 & 11435 \\
\hline$\%$ within area & 1.52 & 93.30 & 3.83 & 1.20 & 0.13 & 0.02 & 100.00 \\
\hline
\end{tabular}

Fisher's Exact =396.220, P-value=0.000; TM: Town Municipality, SM: Sub-district Municipality, SAO: Sub-district Administrative Organization

\section{Results}

A total of 11,432 participants was included in this study. The majorities of population had a low score of risk for CCA (1-4 points; $93.30 \%)$, followed by moderate risk (5 point; $3.80 \%$ ), and no risk ( 0 point; $1.50 \%)$. However, the high scores of risk group for CCA, with 6,7 and 8 points were $1.20 \%, 0.10 \%$ and $0.10 \%$, respectively. The population at risk was classified for 6,7 , and 8 scores, by the type of local administrative organization found that Sub-district Municipality was found frequently, $1.3 \%, 0.10 \%$, and $0.0 \%$ (Table 3). Comparison between the high scores $(6,7$, and 8 points) and type of local administrative organization found that sub-district administrative organization, Sub-district Municipality and Town Municipality were statistically significant different with $\mathrm{F}=396.604$, P-value $=0.000$.

Geovisual display of the population at risk for CCA on

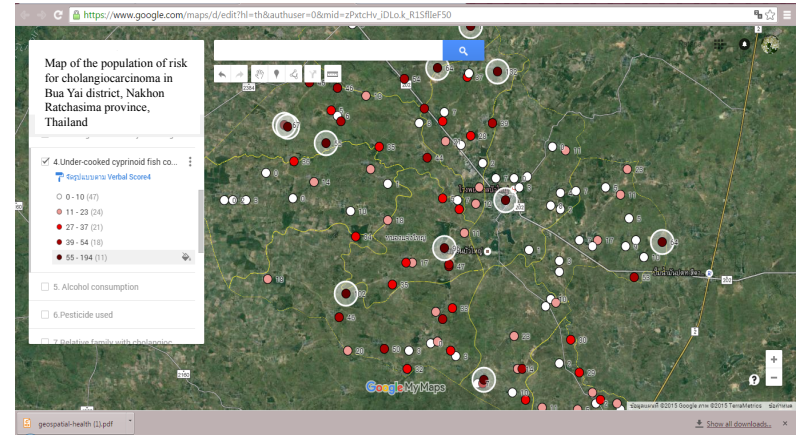

Figure 1. Layer of the Population at risk for CCA in Bua Yai, Nakhon Ratchasima Province Thailand using Google map; https://www.google.com/maps/d/edit?hl $=$ th\&authuser $=0 \&$ mid $=z P x t c H v \_i D L o . k \_R 1 S f l I e F 50$

the GM in this study include 11 layers, for each layer using could be click scroll and choos $\checkmark$; layer 1; district, layer 
2; local administrative organization: Town Municipality, Sub-district Municipality, and Sub-district Administrative Organization, layer 3; hospital, layer 4; KCVST opisthorchiasis, layer 5; KCVST praziquantel used, layer 6; KCVST cholelithiasis, layer 7;KCVST raw fish consumption,layer $8 ; \mathrm{KCVST}$ alcohol consumption,layer 9;KCVST pesticide used, layer 10; KCVST relative family with CCA, and layer 11; KCVST naïve northeastern people (Figure 1).Geovisual display is now available in GM following https://www.google.com/maps/d/edit?hl= th\&authuser=0\&mid=zPxtcHv_iDLo.k_R1SflIeF50

\section{Discussion}

The mortality rate of CCA has been reported that Nakhon Ratchasima province has 13.67-16.2 per 100,000 populations (Sripa et al., 2011). In addition, Opisthorchis viverrini; the carcinogenic liver fluke, also has been reported in this province (Kaewpitoon et al., 2012c; Kaewpitoon et al., 2015). Furthermore, the infective stage of $O$. viverrini was found frequently coverage areas approximately $78.1 \%$ of province (Kaewpitoon et al., 2012a). Base on previously investigated data indicate that Nakhon Ratchasima province is still a problem with this CCA. Here we recent report the population at risk for CCA in Bua Yai district, Nakhon Ratchasima province, Thailand by using KCVST and fed to GM for geovisual display.

Recent study reveals that the majorities of participant had a low score for CCA risk in this district, this figure indicates that a set zero campaign should be further selected. However, $1.40 \%$ of participants had high scores for CCA risk (summarized from 6-8 points), particularly in Sub-district Administrative Organization and Sub-district Municipality. Therefore, monitoring project should be concerned and immediately CCA screened including health behavior changed.

Geovisual display of the population at risk for CCA on the GM including11 layers, this distribution map was developed from the previous study. GM production process is very simple and easy to learn. Distribution point of the population at risk for CCA will display when click scroll and choos $\checkmark$;layer 1 ; district, layer 2 ; local administrative organization: Town Municipality, Subdistrict Municipality, and Sub-district Administrative Organization, layer 3; hospital, layer 4; KCVST opisthorchiasis, layer 5; KCVST praziquantel used, layer 6;KCVST cholelithiasis, layer 7;KCVST raw fish consumption,layer 8;KCVST alcohol consumption,layer 9; KCVST pesticide used, layer 10; KCVST relative family with CCA, and layer 11; KCVST naïve northeastern people. This development tool can be use for further surveillance and monitoring the population at risk in each sub-district, particularly the sub-district where have the high scores of CCA risk. Recent study is similar to previously investigate that GM) production process is very simple and suitable for the user in the further CCA management strategy (Rattanasing et al., 2015). The spatial distribution of risk areas of liver fluke, is an useful tool for future planning of prevention and control measures (Rujirakul et al., 2015).

In conclusion, this recent study indicate that the population at risk for CCA is low level, therefore set zero model project is possible work in this areas, and the key success factor of disease prevention and control is need to further study. However, some areas of sub-districts have a high score of CCA risk, therefore GM production is suitable further CCA surveillance and monitoring in this area.

\section{Acknowledgements}

This work was supported by Suranaree University of Technology (SUT) and by Office of the Higher Education Commission under NRU Project of Thailand. Some part of this work was supported by the National Health Security Office of Nakhon Ratchasima province, through health promotion and prevention fund, year 2015. Special thanks all staffs of district public health organization of Bua Yai for their assistance.

\section{References}

Akamatsu N, Sugawara Y, Hashimoto D (2011). Surgical strategy for bile duct cancer: Advances and current limitations. World J ClinOncol, 10, 94-107.

Bhudhisawasdi V, Khuntikeo N, Chur-in S, et al (2012). Cholangiocarcinoma: experience of Srinagarind Hospital. Srinagarind Med J, 27, 331-9.

Green A, Uttaravichien T, Bhudhisawasdi V, et al (1991). Cholangiocarcinoma in northeast Thailand. A hospital-based study. Trop Geogr Med, 43, 193-8.

Kaewpitoon N, Kaewpitoon SJ, Ueng-arporn N, et al (2012). Carcinogenic human liver fluke: current status of Opisthorchis viverrini metacercariae in Nakhon Ratchasima, Thailand. Asian Pac J Cancer Prev, 13, 1235-40.

Kaewpitoon SJ, Rujirakul R, Kaewpitoon N (2012). Prevalence of Opisthorchis viverrini infection in Nakhon Ratchasima province, Northeast Thailand. Asian Pac J Cancer Prev, 13, 5245-9.

Kaewpitoon N, Kootanavanichpong N, Kompor P, et al (2015). Review and current status of Opisthorchis viverrini infection at the community level in Thailand. Asian Pac J Cancer Prev, 16, 6825-30.

Khuntikao N (2005). Current concept in management of cholangiocarcinoma. Srinagarind Med J, 20, 143-9.

Rattanasing W, Kaewpitoon SJ, Loyd RA, et al (2015). Utilization of Google earth for distribution mapping of cholangiocarcinoma: a case study in Satuek district, Buriram, Thailand. Asian Pac J Cancer Prev, 16, 5903-6.

Rujirakul R, Ueng-arporn N, Kaewpitoon S, et al (2015). GISbased spatial statistical analysis of risk areas for liver flukes in Surin Province of Thailand. Asian Pac J Cancer Prev, 16, 2323-6.

Shin HR, Oh JK, Masuyer E, et al (2010). Epidemiology of cholangiocarcinoma: An update focusing on risk factors. Cancer Sci, 101, 579-585.

Sripa B, Kaewkes S, Sithithaworn P M, et al. (2007). Liver fluke induces cholangiocarcinoma. PLoS Med, 4, 201.

Sripa B, Pairojkul C (2008). Cholangiocarcinoma: lessons from Thailand. Curr Opin Gastroenterol, 24, 349-56.

Sripa B, Bethony JM, Sithithaworn P, et al (2011). Opisthorchiasis and opisthorchis-associated cholangiocarcinoma in Thailand and Laos. Acta Trop, 120, 158-68. 